\title{
Uma Técnica de Expurgo de Limitantes da Probabilidade de Erro de Códigos Espácio-Temporais para Canais com Desvanecimento
}

\author{
Luiz Guedes Caldeira e Cecílio José Lins Pimentel \\ guedes@cefetpb.edu.br - CEFETPB \\ cecilio@ufpe.br - Grupo de Pesquisas em Comunicações - CODEC - Departamento de Eletrônica e Sistemas - UFPE
}

\begin{abstract}
Resumo-Este artigo apresenta uma técnica de expurgo para limitantes da probabilidade de erro de códigos espáciotemporais em treliça em canais com desvanecimentos do tipo Rayleigh e Rice. A técnica apresentada neste artigo é de simples implementação e conforme simulações os limitantes obtidos com esta técnica são muito apertados.
\end{abstract}

Palavras-Chave-Códigos espácio-temporais, limitante da probabilidade de erro.

\section{INTRODUÇÃO}

Considere um esquema de transmissão, conforme a Figura 1, que emprega múltiplas antenas (MIMO - do inglês multiple input multiple output) $\operatorname{com} n_{T}$ antenas de transmissão e $n_{R}$ antenas de recepção, causando uma superposição linear dos $n_{T}$ sinais em cada uma das $n_{R}$ antenas de recepção, devido aos $n_{T} \times n_{R}$ percursos existentes. Considere que a transmissão é realizada em um canal com desvanecimento plano, não seletivo em freqüência, e que os coeficientes do desvanecimento do canal, $h_{i j}, i=1, \ldots, n_{T}, j=1, \ldots, n_{R}$, permaneçam constantes durante a transmissão de um bloco de sinais, de comprimento $L$, transmitidos por cada antena. Considere, ainda, que estes coeficientes mudem independentemente a cada novo bloco transmitido, e que existe espaçamento suficiente entre as antenas de recepção de modo que os sinais transmitidos em cada um dos $n_{T} \times n_{R}$ percursos experimentem desvanecimentos independentes. A expressão do sinal de saída do esquema MIMO, sob tais considerações, pode ser dada na forma matricial:

$$
\mathbf{Y}=\mathbf{H C}+\mathbf{W}
$$

onde $\mathbf{C}$ é a matriz de sinais transmitida, $n_{T} \times L$, cuja energia média da constelação é $E_{s}, \mathbf{H}$ é a matriz $n_{R} \times n_{T}$ dos coeficientes de desvanecimento do canal e $\mathbf{W}$ a matriz de ruído branco, $n_{R} \times L$, com média zero e variância complexa $N_{0}$. Considere ainda que por este canal MIMO são transmitidos códigos espácio-temporais (STTCs - do inglês space time trellis codes) [1]. Considerando que o receptor tenha uma estimativa perfeita do estado do canal, um limitante superior para a probabilidade do primeiro evento erro de um bloco,

Este trabalho recebeu suporte parcial do Conselho Nacional para o Desenvolvimento Científico e Tecnológico (CNPq), No. 300987/96-0.

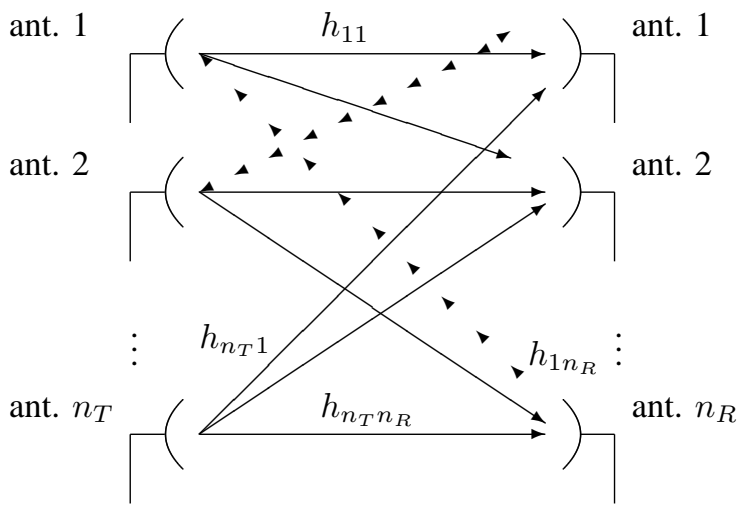

Fig. 1. Esquema MIMO de $n_{T} \times n_{R}$ antenas.

que está diretamente relacionado com a taxa de erro de bloco (FER - do inglês frame error rate), de tal esquema de transmissão empregando STTCs pode ser obtido após uma pequena modificação de (5) em [1] para:

$$
P_{f e \mid \mathbf{H}_{1}^{n_{R}}}(e) \simeq \sum_{i=1}^{|\mathcal{S}|} a_{i} Q\left(\sqrt{\frac{\gamma}{2} \sum_{j=1}^{n_{R}} \mathbf{H}_{j} \mathbf{A}_{i} \mathbf{H}_{j}^{*}}\right),
$$

onde $\mathbf{H}_{1}^{n_{R}}=\left[\mathbf{H}_{1}, \ldots, \mathbf{H}_{n_{R}}\right], \mathbf{H}_{j}=\left[\mathbf{H}_{1, n}, \ldots, \mathbf{H}_{n_{T}, n}\right]$ é um vetor linha dos coeficientes do desvanecimento, $(.)^{*}$ implica em conjugado transposto de $(\cdot), \gamma=E_{s} / N_{0}$ é a relação sinal ruído (SNR - do inglês signal to noise ratio) por antena transmissora e $\mathbf{A}_{i}$ é a matriz Hermitiana do evento erro (EEM do inglês error event matrix) [2] do STTC e $a_{i}$ a sua respectiva multiplicidade. As EEMs dominantes são especificadas por um conjunto $\mathcal{S}$, de cardinalidade $|\mathcal{S}|$. Em [3], o espectro de distâncias de ordem $N$ de um STTC, é a enumeração do conjunto ordenado de pares de determinantes e suas respectivas multiplicidades médias, $\left\{\Delta_{k}, a_{\Delta_{k}}\right\}_{k=1}^{N}$, tal que $\Delta_{k}<\Delta_{k+1}$. Para a aplicação de (2), precisamos enumerar as EEMs. A técnica proposta neste artigo consiste em enumerar, em ordem de importância, as EEMs que dominam o limitante em (2), de modo a obtermos o espectro de distâncias que proporcione um limitante mais apertado possível. 


\section{A. Obtenção das EEMs}

Empregando um algoritmo de programação simbólica [4], foram extraídas várias EEMs para alguns STTCs da literatura. As Tabelas I e II, III e IV, V e VI relacionam, para os códigos de [1], [5] e [6], com 4 e 8 estados respectivamente, os número de estados, índice da EEM, a EEM, a multiplicidade da EEM, o determinante da EEM, e a multiplicidade do determinante, denominados $2^{\nu}, i, \mathbf{A}_{i}, a_{\mathbf{A}_{i}}, \Delta, a_{\Delta}$, respectivamente. Para exemplificar a indexação do índice $i$ à matriz $\mathbf{A}_{i}$, ilustraremos o caso da terceira linha da Tabela III: $i=$ $5-8 ;[6,8, \pm 2 \pm j 6]$, que corresponderia às matrizes $\mathbf{A}_{5}=$ $[6,8,2+j 6], \mathbf{A}_{6}=[6,8,2-j 6], \mathbf{A}_{7}=[6,8,-2+j 6], \mathbf{A}_{8}=$ $[6,8,-2-j 6]$, sempre nesta ordem.

\section{TABELA I}

ESPECTRO DOS SEIS PRIMEIROS DETERMINANTES PARA O STTC DE [1], QPSK, COM 4 ESTADOS.

\begin{tabular}{|c|c|c|c|c|c|}
\hline $2^{\nu}$ & $\mathrm{i}$ & $\mathbf{A}_{i}$ & $a_{\mathbf{A}_{i}}$ & $\Delta$ & $a_{\Delta}$ \\
\hline \multirow{6}{*}{4} & 1 & {$[2,2,0]$} & 2 & 4 & 2 \\
\cline { 2 - 6 } & $2-3$ & {$[4,4, \pm 2]$} & 1 cada & 12 & 4 \\
& $4-5$ & {$[4,4, \pm j 2]$} & 1 cada & & \\
\cline { 2 - 6 } & 6 & {$[4,4,0]$} & 1 & 16 & 1 \\
\cline { 2 - 6 } & $7-8$ & {$[6,6, \pm 4]$} & $1 / 2$ cada & 20 & 2 \\
& $9-10$ & {$[6,6, \pm j 4]$} & $1 / 2$ cada & & \\
\cline { 2 - 7 } & $11-14$ & {$[6,6, \pm 2 \pm j 2]$} & 2 cada & 28 & 9 \\
& $15-16$ & {$[8,8, \pm j 6]$} & $1 / 4$ cada & & \\
& $17-18$ & {$[8,8, \pm 6]$} & $1 / 4$ cada & & \\
\cline { 2 - 7 } & $19-22$ & {$[8,8, \pm 4 \pm j 4]$} & $1 / 4$ cada & 32 & 1 \\
\hline
\end{tabular}

TABELA II

ESPECTRO QUATRO PRIMEIROS DETERMINANTES PARA O STTC DE [1], QPSK, COM 8 ESTADOS.

\begin{tabular}{|c|c|c|c|c|c|}
\hline $2^{\nu}$ & $\mathrm{i}$ & $\mathbf{A}_{i}$ & $a_{\mathbf{A}_{i}}$ & $\Delta$ & $a_{\Delta}$ \\
\hline 8 & $1-4$ & {$[2,10, \pm 2 \pm j 2]$} & $1 / 2$ cada & 12 & 2 \\
\hline & 5 & {$[4,4,0]$} & 1 & 16 & 1 \\
\cline { 2 - 6 } & $6-7$ & {$[6,6, \pm 4]$} & $1 / 2$ cada & 20 & 4 \\
& $8-9$ & {$[6,6, \pm j 4]$} & $1 / 2$ cada & & \\
& 10 & {$[10,2,0]$} & 2 & & \\
\cline { 2 - 6 } & $11-12$ & {$[4,8, \pm 2]$} & 1 cada & 28 & $21 / 2$ \\
& $13-14$ & {$[4,8, \pm j 2]$} & 1 cada & & \\
& $15-18$ & {$[6,6, \pm 2 \pm j 2]$} & $1 / 2$ cada & & \\
& $19-22$ & {$[8,12, \pm 8 \pm j 2]$} & $1 / 16$ cada & & \\
& $23-26$ & {$[8,12, \pm 2 \pm j 8]$} & $1 / 16$ cada & & \\
& $27-28$ & {$[8,4, \pm 2]$} & 1 cada & & \\
& $29-30$ & {$[8,4, \pm j 2]$} & 1 cada & & \\
\hline
\end{tabular}

\section{B. Estatísticas das EEMs}

Com o objetivo de determinar a ordem de importância de cada EEM no espectro de distâncias de um STTC, foi realizado através de simulação da Equação (2), a estatística para cada uma das EEMs de cada STTC. O limitante expurgado da união em (2), associado a uma técnica modificada de limitação e a técnica de calcular a média antes de limitar foi aplicada em [3] para calcular um limitante expurgado da união o mais próximo possível da FER em termos do espectro de distâncias. Isto resultou em:

$$
\mathbf{F E R} \simeq \mathbf{E}\left[f\left(\min \left(1, P_{f e}(e \mid h)\right)\right)\right],
$$

onde $f(x)=1-(1-x)^{L}$, sendo $L$ o comprimento do bloco transmitido e $\mathbf{E}[\alpha]$ o valor esperado da variável aleatória $\alpha$.
TABELA III

ESPECTRO DOS SEIS PRIMEIROS DETERMINANTES PARA O STTC DE [5], QPSK COM 4 ESTADOS.

\begin{tabular}{|c|c|c|c|c|c|}
\hline $2^{\nu}$ & $\mathrm{i}$ & $\mathbf{A}_{i}$ & $a_{\mathbf{A}_{i}}$ & $\Delta$ & $a_{\Delta}$ \\
\hline \multirow{6}{*}{4} & $1-2$ & {$[6,2, \pm j 2]$} & $1 / 2$ cada & 8 & $\frac{3}{2}$ \\
& $3-4$ & {$[6,8, \pm 6-j 2]$} & $1 / 8$ cada & & \\
& $5-8$ & {$[6,8, \pm 2 \pm j 6]$} & $1 / 16$ cada & & \\
\cline { 2 - 7 } & $9-10$ & {$[2,8, \pm 2]$} & $1 / 2$ cada & 12 & $\frac{17}{8}$ \\
& $11-14$ & {$[2,8, \pm 2 \pm j 2]$} & $1 / 4$ cada & & \\
& $15-16$ & {$[8,10, \pm 8+j 2]$} & $1 / 16$ cada & & \\
\cline { 2 - 7 } & $17-20$ & {$[4,6, \pm 2 \pm j 2]$} & $1 / 4$ cada & 16 & 1 \\
\cline { 2 - 7 } & $21-24$ & {$[10,4, \pm 2 \pm j 4]$} & $1 / 8$ cada & 20 & 1 \\
& $25-28$ & {$[10,6, \pm 2 \pm j 6]$} & $1 / 16$ cada & & \\
& $29-32$ & {$[10,6, \pm 6 \pm j 2]$} & $1 / 16$ cada & & \\
\cline { 2 - 7 } & $33-36$ & {$[8,4, \pm 2 \pm j 2]$} & $1 / 4$ cada & 24 & $\frac{3}{2}$ \\
& $37-40$ & {$[8,8, \pm 2 \pm j 6]$} & $1 / 16$ cada & & \\
& $41-44$ & {$[8,8, \pm 6 \pm j 2]$} & $1 / 16$ cada & & \\
\cline { 2 - 7 } & $45-46$ & {$[4,12, \pm 4-j 2]$} & $3 / 8$ cada & 28 & $\frac{77}{32}$ \\
& $47-48$ & {$[8,6, \pm 4-j 2]$} & $1 / 4$ cada & & \\
& $49-50$ & {$[4,12, \pm 4+j 2]$} & $1 / 8$ cada & & \\
& $51-52$ & {$[8,8, \pm 6]$} & $1 / 8$ cada & & \\
& $53-56$ & {$[12,4, \pm 4 \pm j 2]$} & $1 / 8$ cada & & \\
& $57-60$ & {$[8,10, \pm 4 \pm j 6]$} & $1 / 32$ cada & & \\
& $61-64$ & {$[12,12, \pm 4 \pm j 10]$} & $1 / 128$ cada & & \\
\hline
\end{tabular}

TABELA IV

ESPECTRO DOS QUATRO PRIMEIROS DETERMINANTES PARA O STTC DE [5], QPSK COM 8 ESTADOS.

\begin{tabular}{|c|c|c|c|c|c|}
\hline $2^{\nu}$ & $\mathrm{i}$ & $\mathbf{A}_{i}$ & $a_{\mathbf{A}_{i}}$ & $\Delta$ & $a_{\Delta}$ \\
\hline 8 & $1-4$ & {$[4,6, \pm 2 \pm j 2]$} & $1 / 4$ cada & 16 & 1 \\
\cline { 2 - 6 } & $5-8$ & {$[10,10, \pm 4 \pm j 8]$} & $1 / 64$ cada & 20 & $\frac{29}{8}$ \\
& $9-12$ & {$[10,10, \pm 8 \pm j 4]$} & $1 / 64$ cada & & \\
& $13-16$ & {$[10,4, \pm 4 \pm j 2]$} & $1 / 8$ cada & & \\
& $17-20$ & {$[10,4, \pm 2 \pm j 4]$} & $1 / 8$ cada & & \\
& $21-24$ & {$[10,6, \pm 6 \pm j 2]$} & $1 / 16$ cada & & \\
& $25-28$ & {$[10,6, \pm 2 \pm j 6]$} & $1 / 16$ cada & & \\
& 29 & {$[2,10,0]$} & 1 & & \\
& $30-31$ & {$[2,12, \pm j 2]$} & $1 / 2$ cada & & \\
\cline { 2 - 6 } & $32-35$ & {$[12,8, \pm 2 \pm j 8]$} & $1 / 32$ cada & 28 & \multirow{3}{4}{} \\
& $36-39$ & {$[12,8, \pm 8 \pm j 2]$} & $1 / 32$ cada & & \\
& $40-43$ & {$[12,4, \pm 2 \pm j 4]$} & $1 / 8$ cada & & \\
\cline { 2 - 6 } & $44-47$ & {$[8,8, \pm 4 \pm j 4]$} & $1 / 16$ cada & 32 & $\frac{47}{16}$ \\
& $48-49$ & {$[6,8, \pm j 4]$} & $1 / 4$ cada & & \\
& $50-51$ & {$[6,8, \pm 4]$} & $1 / 4$ cada & & \\
& $52-53$ & {$[6,6, \pm j 2]$} & $1 / 2$ cada & & \\
& $54-57$ & {$[14,12, \pm 10 \pm j 6]$} & $1 / 256$ cada & & \\
& $58-61$ & {$[14,12, \pm 6 \pm j 10]$} & $1 / 256$ cada & & \\
& $62-65$ & {$[14,6, \pm 4 \pm j 6]$} & $1 / 32$ cada & & \\
& $66-69$ & {$[14,6, \pm 6 \pm j 4]$} & $1 / 32$ cada & & \\
& $70-73$ & {$[12,14, \pm 10 \pm j 6]$} & $1 / 256$ cada & & \\
& $74-77$ & {$[12,14, \pm 6 \pm j 10]$} & $1 / 256$ cada & & \\
$78-81$ & {$[12,6, \pm 6 \pm j 2]$} & $1 / 16$ cada & & \\
& $82-85$ & {$[14,8, \pm 4 \pm j 8]$} & $1 / 64$ cada & & \\
& $86-89$ & {$[14,8, \pm 8 \pm j 4]$} & $1 / 64$ cada & & \\
\hline
\end{tabular}

Em [3] foi observado que uma boa precisão do limitante é alcançada com um espectro de distâncias composto dos primeiros determinantes, não necessariamente os menores. A técnica que propomos analisa a importância individual de cada EEM e não uma classe de EEMs com mesmo determinante.

Considerando a ordem de dominância de uma variável aleatória, propomos neste trabalho uma metodologia para identificar o conjunto dominante de EEMs que proporciona um limitante apertado. Seja $P^{i}\left(\mathbf{H}_{1}^{n_{R}}\right)$ a contribuição de cada 
TABELA V

ESPECTRO DOS SEIS PRIMEIROS DETERMINANTES PARA O STTC DE [6], QPSK, COM 4 ESTADOS.

\begin{tabular}{|c|c|c|c|c|c|}
\hline $2^{\nu}$ & $\mathrm{i}$ & $\mathbf{A}_{i}$ & $a_{\mathbf{A}_{i}}$ & $\Delta$ & $a_{\Delta}$ \\
\hline \multirow{6}{*}{4} & $1-4$ & {$[6,6, \pm 4 \pm j 4]$} & $1 / 16$ cada & 4 & $\frac{1}{4}$ \\
\cline { 2 - 6 } & $5-8$ & {$[8,2, \pm 2 \pm j 2]$} & $1 / 4$ cada & 8 & 2 \\
& $9-12$ & {$[2,8, \pm 2 \pm j 2]$} & $1 / 4$ cada & & \\
\cline { 2 - 6 } & $13-16$ & {$[8,8, \pm 4 \pm j 6]$} & $1 / 32$ cada & 12 & \\
\cline { 2 - 6 } & $17-20$ & {$[6,8, \pm 4 \pm j 4]$} & $1 / 16$ cada & 16 & $\frac{1}{2}$ \\
& $21-24$ & {$[8,6, \pm 4 \pm j 4]$} & $1 / 16$ cada & & \\
\cline { 2 - 6 } & $25-26$ & {$[6,6, \pm 4]$} & $1 / 8$ cada & 20 & $\frac{27}{16}$ \\
& $27-28$ & {$[6,6, \pm j 4]$} & $1 / 8$ cada & & \\
& $29-32$ & {$[10,4, \pm 4 \pm j 2]$} & $1 / 8$ cada & & \\
& $33-36$ & {$[4,10, \pm 4 \pm j 2]$} & $1 / 8$ cada & & \\
& $37-40$ & {$[10,10, \pm 8 \pm j 4]$} & $1 / 64$ cada & & \\
& $41-44$ & {$[10,10, \pm 4 \pm j 8]$} & $1 / 64$ cada & & \\
\cline { 2 - 7 } & $45-48$ & {$[4,8, \pm 2 \pm j 2]$} & $1 / 4$ cada & 24 & 2 \\
& $49-52$ & {$[8,4, \pm 2 \pm j 2]$} & $1 / 4$ cada & & \\
\hline
\end{tabular}

TABELA VI

ESPECTRO DOS SETE PRIMEIROS DETERMINANTES PARA O STTC DE [6], QPSK, COM 8 ESTADOS.

\begin{tabular}{|c|c|c|c|c|c|}
\hline $2^{\nu}$ & $\mathrm{i}$ & $\mathbf{A}_{i}$ & $a_{\mathbf{A}_{i}}$ & $\Delta$ & $a_{\Delta}$ \\
\hline 8 & $1-4$ & {$[8,6, \pm 6 \pm j 2]$} & $1 / 16$ cada & 8 & $\frac{1}{2}$ \\
\cline { 2 - 6 } & $5-8$ & {$[8,6, \pm 2 \pm j 6]$} & $1 / 16$ cada & & \\
\cline { 2 - 6 } & $9-12$ & {$[10,2, \pm 2 \pm j 2]$} & $1 / 4$ cada & 12 & 1 \\
\cline { 2 - 6 } & $13-14$ & {$[2,10, \pm 2]$} & $1 / 2$ cada & 16 & 1 \\
\cline { 2 - 6 } & $15-18$ & {$[8,8, \pm 6 \pm j 2]$} & $1 / 16$ cada & 24 & $\frac{1}{2}$ \\
& $19-22$ & {$[8,8, \pm 2 \pm j 6]$} & $1 / 16$ cada & & \\
\cline { 2 - 6 } & $23-26$ & {$[6,8, \pm 4 \pm j 2]$} & $1 / 8$ cada & 28 & $\frac{1}{2}$ \\
\cline { 2 - 6 } & $27-30$ & {$[8,6, \pm 2 \pm j 2]$} & $1 / 8$ cada & 40 & $\frac{3}{2}$ \\
\cline { 2 - 7 } & $31-34$ & {$[6,8, \pm 2 \pm j 2]$} & $1 / 4$ cada & & \\
\cline { 2 - 6 } & $35-36$ & {$[6,8, \pm j 2]$} & $1 / 4$ cada & 44 & $\frac{1}{2}$ \\
\hline
\end{tabular}

matriz $\mathbf{A}_{i}$ para $P_{f e \mid \mathbf{H}_{1}^{n_{R}}}(e)$ :

$$
P^{i}\left(\mathbf{H}_{1}^{n_{R}}\right)=a_{i} Q\left(\sqrt{\frac{\gamma}{2} \sum_{j=1}^{n_{R}} \mathbf{H}_{j} \mathbf{A}_{i} \mathbf{H}_{j}^{*}}\right) .
$$

Seja $\mathcal{A}\left(\mathbf{H}_{1}^{n_{R}}\right)=\left\{\mathbf{A}_{j} 1, \mathbf{A}_{j} 1, \ldots, \mathbf{A}_{j} k, \ldots\right\}$ ser o conjunto de EEMs tal que $P^{j k}\left(\mathbf{H}_{1}^{n_{R}}\right) \geq P^{j k+1}\left(\mathbf{H}_{1}^{n_{R}}\right)$. Defina uma variável aleatória $X_{i}$ como a posição da matriz $\mathbf{A}_{i}$ em $\mathcal{A}\left(\mathbf{H}_{1}^{n_{R}}\right)$. Esta variável aleatória é uma função dos coeficientes de desvanecimento $\left(\mathbf{H}_{1}^{n_{R}}\right)$. Utilizaremos o valor esperado de $X_{i}$, denominado $\bar{X}_{i}=\mathbf{E}\left[X_{i}\right]$, para ordenar as matrizes em ordem decrescente de dominância. As Tabelas VII, VIII e IX ilustram as classificações das EEMs, obtidas através de simulações, para os STTCs de [1], [5] e [6], respectivamente, para o canal Rayleigh. As Tabelas X, XI e XII ilustram as classificações das EEMs destes mesmos STTCs para o canal Rice.

Os valores distintos de $\bar{X}_{i}$, ordenados de forma crescente formam o conjunto ordenado $\beta^{\iota}=\left\{\beta_{k}\right\}_{k=1}^{\iota} \operatorname{com} \iota$ elementos, onde $\beta_{k}<\beta_{k+1}$. Por exemplo, a partir dos valores listados na Tabela VII para o STTC de 4 estados, obtemos $\beta^{6}=$ $\{1,0 ; 6,0 ; 6,1 ; 10,4 ; 11,1 ; 13,2\}$. Seja $\mathcal{S}^{\iota}$ o conjunto de todas as EEMs $\mathbf{A}_{i}$ tal que $\bar{X}_{i} \in \beta^{\iota}$. A FER expurgada obtida com as EEMs em $\mathcal{S}^{\iota}$ é denotada por FER ${ }^{\iota}$.

As condições utilizadas para as simulações das estatísticas das EEMs para ambos os modelos de canais foram: $\mathrm{SNR}=15$ $\mathrm{dB}$, blocos de comprimento $L=130, n_{R}=1$. No caso do canal Rice, foi empregado um fator de Rice $K_{r}=3 \mathrm{~dB}$.

TABELA VII

$\bar{X}_{i}$ PaRa os STTCS Das Tabelas I, II. CANal RaYleigh.

\begin{tabular}{|c|c||c|c|}
\hline i (4 estados) & $\bar{X}_{i}$ & i (8 estados) & $\bar{X}_{i}$ \\
\hline 1 & 1.0 & 5 & 6.3 \\
\hline $2-5$ & 6 & $1-4$ & 13.7 \\
\hline 6 & 6.1 & $6-18$ & 13.8 \\
\hline $7-10$ & 10.4 & $27-30$ & 14.0 \\
\hline $11-14$ & 11.1 & $19-26$ & 20.8 \\
\hline $15-18$ & 13.2 & & \\
\hline
\end{tabular}

TABELA VIII

$\bar{X}_{i}$ PARA os STTCS Das TABElas III, IV. CANAL RaYleigh.

\begin{tabular}{|c|c||c|c|}
\hline i (4 estados) & $X_{i}$ & i (8 estados) & $X_{i}$ \\
\hline $1-2$ & 14 & $1-4$ & 22.1 \\
\hline $9-10,17-20$ & 20.3 & $52-53$ & 28.1 \\
\hline $33-36$ & 25.7 & 29 & 30.6 \\
\hline $11-14$ & 26.4 & $13-20$ & 32.7 \\
\hline $3-8,47-48$ & 30.6 & $48-51$ & 34.5 \\
\hline $21-24$ & 31.1 & $30-31$ & 36.5 \\
\hline $25-29,31,37,39$ & 35.5 & $21-28$ & 38.4 \\
\hline $41,43,45,46,51-56$ & 35.5 & $40-47$ & 39.5 \\
\hline $30,32,38,40$ & 36.1 & $78-81$ & 44.8 \\
\hline $42,44,49-50$ & 36.1 & $6-12$ & 48.1 \\
\hline $15-16$ & 38.9 & $32-39$ & 49.1 \\
\hline $57-60$ & 39.8 & $62-69$ & 49.5 \\
\hline $61-64$ & 46.7 & $82-89$ & 53.6 \\
\hline & & $70-77$ & 59 \\
\hline & & $54-61$ & 59.4 \\
\hline
\end{tabular}

TABELA IX

$\bar{X}_{i}$ PaRa os STtCs das Tabelas V, VI. Canal Rayleigh.

\begin{tabular}{|c|c||c|c|}
\hline i (4 estados) & $\bar{X}_{i}$ & i (8 estados) & $\bar{X}_{i}$ \\
\hline $5-12$ & 18.4 & $13-14$ & 14.6 \\
\hline $1-4$ & 22.5 & $9-12$ & 15.2 \\
\hline $25-28,45-52$ & 23.3 & $31-36$ & 17.9 \\
\hline $17-24,29-36$ & 27.8 & $23-26$ & 18.3 \\
\hline $13-16$ & 31.0 & $1-8$ & 18.4 \\
\hline $37-44$ & 36.4 & $27-30$ & 18.5 \\
\hline & & $19-22$ & 21.8 \\
\hline & & $15-18$ & 22 \\
\hline
\end{tabular}

TABELA X

$\bar{X}_{i}$ PaRa os STtCs das Tabelas I, II. Canal Rice.

\begin{tabular}{|c|c||c|c|}
\hline i (4 estados) & $X_{i}$ & i (8 estados) & $X_{i}$ \\
\hline 1 & 1.0 & 7 & 3.6 \\
\hline 3 & 2.8 & 5 & 6.3 \\
\hline 8 & 5.5 & 21,22 & 7.8 \\
\hline 4,5 & 6.2 & $12,17,18,28$ & 8.5 \\
\hline 6 & 6.4 & 3,4 & 9.1 \\
\hline 18 & 7 & $8-10,13,14,29,30$ & 14 \\
\hline 13,14 & 9 & 1,2 & 19 \\
\hline 2 & 9.6 & $11,15,16,27$ & 19.5 \\
\hline 9,10 & 10.8 & 25,26 & 19.8 \\
\hline 15,16 & 13.3 & 6 & 23.8 \\
\hline 11,12 & 13.7 & 23,24 & 25.1 \\
\hline 7 & 15.1 & 19,20 & 28.2 \\
\hline 17 & 17.2 & & \\
\hline
\end{tabular}


TABELA XI

$\bar{X}_{i}$ PaRa os STTCS das Tabelas III, IV. CANAl Rice.

\begin{tabular}{|c|c|c|c|}
\hline i (4 estados) & $\overline{X_{i}}$ & i (8 estados) & $X_{i}$ \\
\hline 2 & 6.3 & 23,24 & 12.2 \\
\hline 4 & 7.7 & 3,4 & 13 \\
\hline hline 16 & 10.7 & 13,14 & 13.6 \\
\hline $10,19,20,52$ & 12.5 & 15,16 & 15.7 \\
\hline $31,32,43,44$ & 13,2 & 51 & 16.4 \\
\hline 48 & 15.7 & 38,39 & 17 \\
\hline 35,36 & 18.5 & 11,12 & 17.3 \\
\hline 13,14 & 18.9 & 80,81 & 20.5 \\
\hline 55,56 & 21.5 & 46,47 & 23.5 \\
\hline 46,50 & 22.2 & 88,89 & 24.1 \\
\hline 7,8 & 23.5 & 19,20 & 26 \\
\hline $1,23,24$ & 24 & 68,69 & 27.2 \\
\hline 59,60 & 27 & $56,57,72,73$ & 27.7 \\
\hline 27,28 & 29.2 & $52,53,29$ & 29.3 \\
\hline $9,17,18,39,40$ & 29.5 & 27,28 & 32.5 \\
\hline 33,34 & 34.5 & 42,43 & 33 \\
\hline 11,12 & 34.9 & 1,2 & 33.2 \\
\hline $5,6,21,22$ & 38.7 & 7,8 & 35.5 \\
\hline 63,64 & 39.1 & $30,31,48,49,64,65$ & 36.2 \\
\hline 25,26 & 43.2 & 84,85 & 42 \\
\hline 37,38 & 43.6 & 17,18 & 43.8 \\
\hline 47 & 45.3 & $76,77,60,61$ & 44.4 \\
\hline $45,53,54$ & 48.7 & 34,35 & 44.7 \\
\hline 49 & 49 & $40,41,25,26$ & 49.4 \\
\hline 41,42 & 50.2 & 50 & 51.7 \\
\hline 3 & 51.1 & 44,45 & 56.5 \\
\hline 57,58 & 52.3 & 32,33 & 58 \\
\hline 29,30 & 54.5 & 21,22 & 62.5 \\
\hline 51 & 55.2 & $5,6,62,63$ & 63.9 \\
\hline 61,62 & 57 & 78,79 & 66.8 \\
\hline \multirow[t]{9}{*}{15} & 60.7 & 82,83 & 68 \\
\hline & & 66,67 & 70.3 \\
\hline & & 9,10 & 74.8 \\
\hline & & 36,37 & 75.4 \\
\hline & & 74,75 & 76.3 \\
\hline & & 58,59 & 76.7 \\
\hline & & 86,87 & 78.1 \\
\hline & & 70,71 & 83.7 \\
\hline & & 54,55 & 84.1 \\
\hline
\end{tabular}

TABELA XII

$\bar{X}_{i}$ PaRa os STTCS Das TABElas V, VI. CANAL Rice.

\begin{tabular}{|c|c||c|c|}
\hline i (4 estados) & $X_{i}$ & i (8 estados) & $X_{i}$ \\
\hline 26 & 9 & 3,4 & 9.6 \\
\hline 3,4 & 9.5 & $14,25,26$ & 12 \\
\hline $7,8,11,12$ & 11.8 & 11,12 & 12.3 \\
\hline $19,20,23,24,31,32,35,36$ & 15.2 & 17,18 & 13 \\
\hline 39,40 & 15.5 & 33,34 & 15 \\
\hline $47,48,51,52$ & 17.6 & $7,8,29,30$ & 15.35 \\
\hline 15,16 & 20.5 & $9,10,13,35,36$ & 18.2 \\
\hline 27,28 & 24.6 & 21,22 & 18.8 \\
\hline $5,6,9,10$ & 26 & 5,6 & 21.2 \\
\hline 43,44 & 28.4 & 31,32 & 21.4 \\
\hline $45,46,49,50$ & 30.6 & 27,28 & 21.8 \\
\hline 1,2 & 35.7 & $19,20,23,24$ & 24.5 \\
\hline 25 & 37 & 1,2 & 26.7 \\
\hline $17,18,21,22,29,30,33,34$ & 40 & 15,16 & 29.7 \\
\hline 13,14 & 42.3 & & \\
\hline 41,42 & 45.7 & & \\
\hline 37,38 & 50.1 & & \\
\hline & & &
\end{tabular}

\section{REsultados}

Para analisarmos melhor o valor apropriado de $\iota$ tal que FER $^{\iota}$ aproxima a FER obtida por simulação, exemplificaremos o caso da FER parcial do STTC [1], 8 estados, para o canal
TABELA XIII

EEMS UTILIZADAS NAS SIMULAÇÕES PARA OS STTCS [1], [5] E [6], DE 4 E 8 ESTADOS, PARA O CANAL RAYLEIGH (VER TABELAS I, II E III).

\begin{tabular}{|c|c|c|}
\hline STTC & $2^{\nu}$ & Índice $i$ das EEMs $\mathbf{A}_{i}$ \\
\hline \multirow{2}{*}[1]{} & 4 & $1-5$ \\
\cline { 2 - 3 } & 8 & $1-14$ \\
\hline \multirow{2}{*}[5]{} & 4 & $1,2,9,10,17-20$ \\
\cline { 2 - 3 } & 8 & $1-4,13-20,29-31,48-53$ \\
\hline \multirow{2}{*}[6]{} & 4 & $1-12$ \\
\cline { 2 - 3 } & 8 & $9-14,31-36$ \\
\hline
\end{tabular}

TABELA XIV

EEMs UTILIZADAS NAS SIMULAÇÕES PARA OS STTCS [1], [5] E [6] PARA O CANAL RICE (VER TABELAS I, II E III).

\begin{tabular}{|c|c|c|}
\hline STTC & $2^{\nu}$ & Índice $i$ das EEMs $\mathbf{A}_{i}$ \\
\hline \multirow{2}{*}[1]{} & 4 & $1,3-6,8$ \\
\cline { 2 - 3 } & 8 & $1-5,7-18,21,22,27-30$ \\
\hline$[5]$ & 4 & $2,4,10,16,19,20,31,32,35,36,43,44,48,52$ \\
\cline { 2 - 3 } & 8 & $1-4,7,8,11-20,23-31,34,35,38-43,46-49$, \\
& & $51-53,56,57,60,61,64,65,68,69,72,73,76$, \\
& & $77,80,81,84,85,88,89$ \\
\hline$[6]$ & 4 & $3,4,7,8,11,12,19,20,23,24,26,31,32,35,36$ \\
\cline { 2 - 3 } & 8 & $3,4,7-14,17,18,25,26,29,30,33-36$ \\
\hline
\end{tabular}

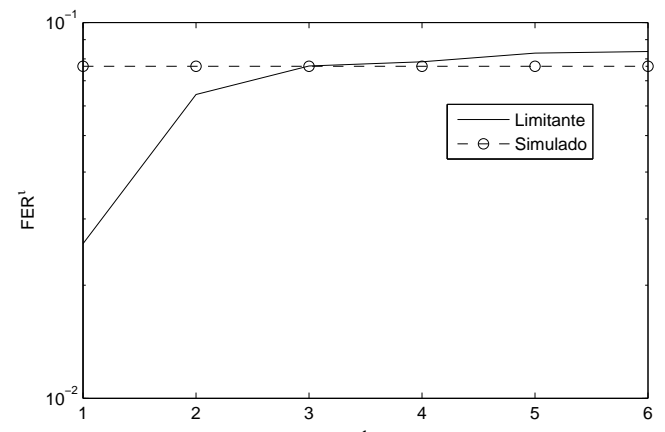

Fig. 2. FER $^{\iota}$ versus $\iota$, para o STTC 8 estados de [1], canal Rayleigh.

Rayleigh. A Figura 2 ilustra resultados numéricos da $\mathrm{FER}^{\iota}$ versus $\iota$, obtidos a partir de (3) usando as EEMs com valores de $\beta^{\iota}$ listados na Tabela VII. Observa-se que o conjunto de médias $\beta^{4}=\{6.3 ; 13.7 ; 13.8 ; 14.0\}$, resulta em $\mathrm{FER}^{4}$, um limitante superior, enquanto que o conjunto formado por $\mathrm{FER}^{3}$ é um limitante inferior. Podemos verificar que estes limitantes são apertados. As Tabelas XIII e XIV listam todas as EEMs utilizadas na simulação dos limitantes expurgados dos STTCs utilizados neste trabalho para os canais Rayleigh e Rice, respectivamente.

As Figuras 3 e 4 ilustram as comparações dos limitantes expurgados com as simulações, para $n_{R}=1,2$, dos STTCs de 4 e 8 estados de [1], para os canais Rayleigh e Rice, respectivamente.

As Figuras 5 e 6 ilustram as simulações dos STTCs de 4 e 8 estados, para o canal Rice, dos códigos de [5] e [6], respectivamente.

\section{CONCLUSÕES}

Neste artigo foi mostrado uma técnica para obtenção de um limitante apertado para STTCs. Esta técnica que consiste na enumeração das EEMs do STTC, em uma ordem 
de importância que contribua de forma mais significativa para (4), difere basicamente da técnica empregada em outros trabalhos [2] por não enumerar as EEMs pelo critério do determinante, pois em alguns códigos a FER parcial não apresenta um bom ajuste do limitante.

Através de simulação semi-analítica foram apresentadas as FERs parciais e posteriormente comparadas ao limitante da união expurgado, com o critério de expurgo obtido em (4), onde expurgava-se a EEM de menor significância na média (maior média), foi também apresentada a simulação do STTC. Uma grande vantagem da técnica proposta é que sempre conseguimos um limitante bem apertado incluindo e/ou expurgando EEMs. Neste artigo foram testados códigos clássicos da literatura, códigos que foram construídos de forma heurística [1], com busca exaustiva [5] e com o critério de maximização da distância Euclidiana [6]. Mesmo empregando códigos com características de construção diferentes, o emprego da técnica proposta teve sucesso. A técnica empregada mostrou-se eficiente para ambos os tipos de desvanecimento, Rayleigh e Rice.

\section{REFERÊNCIAS}

[1] V. Tarokh,N. Seshadri and A.R. Calderbank, Space-Time Codes for high data rate wireless communication: Performance criteria and code construction. IEEE Transactions of Information Theory, v. 44, p. 744764, March, 1998

[2] D.K. Aktas and M. P. Fitz, The distance spectrum of space-time trellis coded modulations in quasi-static Rayleigh fading channels. IEEE Transactions of Information Theory, v. 49, p. 3335-3344, 2003.

[3] A. P. des Rosiers and P.H. Siegel, Space-Time code performance bounds on quasistatic fading channels. in Proc. IEEE ICC'2003, Anchorage, USA, 2003, p. 3160-3164.

[4] Luiz G. Caldeira and Cecilio J. L. Pimentel, An Iterative MatrixBased Algorithm to Finding the Distance Spectrum of Space-Time Trellis Codes. International Symposium of Information Theory and Applications - ISITA 2004, Parma, Italy, october 2004, p. 331-334.

[5] Q. Yan and R. S. Blum, Optimum Space-Time Codes. IEEE Wireless Comm. and Networking Conf., Chicago, USA, September 2000, p. 13511355.

[6] Zhuo Chen, Jinhong Yuan and Branka Vucetic, An Improved Space-Time Trellis Coded Modulation Scheme on Slow Rayleigh Fading Channels. IEEE International Conference on Communication, ICC 2001, no. 1, June 2001, p. 1110-1116.

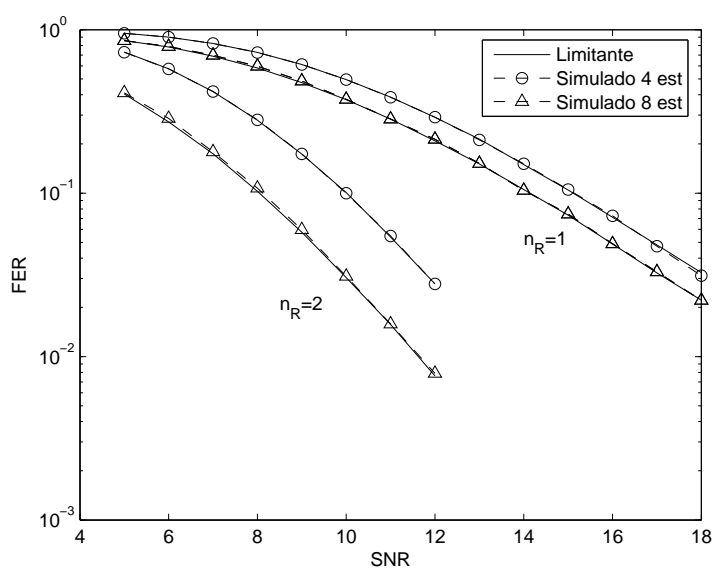

Fig. 3. FER versus SNR por antena recebida para os STTCs de 4 e 8 estados de [1], $n_{R}=1,2$ e canal Rayleigh.

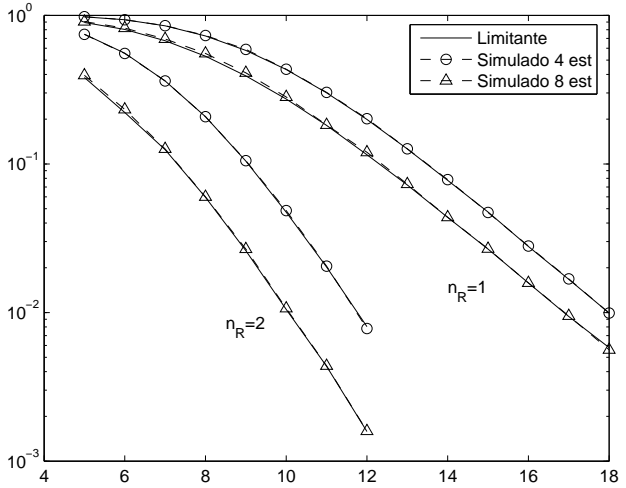

Fig. 4. FER versus SNR por antena recebida para os STTCs de 4 e 8 estados de [1], $n_{R}=1,2$ e canal Rice.

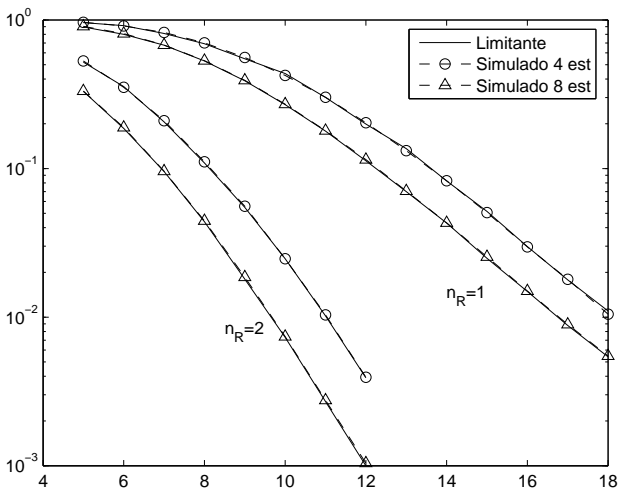

Fig. 5. FER versus SNR por antena recebida para os STTCs de 4 e 8 estados de [5], $n_{R}=1,2$, canal Rice.

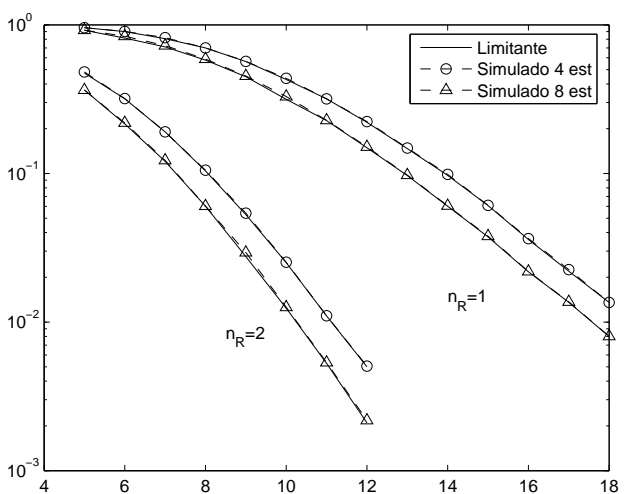

Fig. 6. FER versus SNR por antena recebida para os STTCs de 4 e 8 estados de [6], $n_{R}=1,2$, canal Rice. 